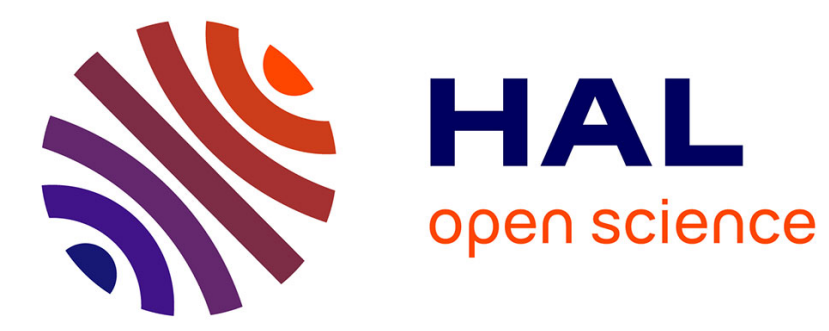

\title{
Real-Time In Situ Observations Reveal a Double Role for Ascorbic Acid in the Anisotropic Growth of Silver on Gold
}

Kinanti Aliyah, Jieli Lyu, Claire Goldmann, Thomas Bizien, Cyrille Hamon, Damien Alloyeau, Doru Constantin

\section{To cite this version:}

Kinanti Aliyah, Jieli Lyu, Claire Goldmann, Thomas Bizien, Cyrille Hamon, et al.. Real-Time In Situ Observations Reveal a Double Role for Ascorbic Acid in the Anisotropic Growth of Silver on Gold. Journal of Physical Chemistry Letters, 2020, 11 (8), pp.2830-2837. 10.1021/acs.jpclett.0c00121 . hal-02997235

\section{HAL Id: hal-02997235 \\ https://hal.science/hal-02997235}

Submitted on 10 Nov 2020

HAL is a multi-disciplinary open access archive for the deposit and dissemination of scientific research documents, whether they are published or not. The documents may come from teaching and research institutions in France or abroad, or from public or private research centers.
L'archive ouverte pluridisciplinaire HAL, est destinée au dépôt et à la diffusion de documents scientifiques de niveau recherche, publiés ou non, émanant des établissements d'enseignement et de recherche français ou étrangers, des laboratoires publics ou privés. 


\title{
Real-Time In SituObservations Reveal a Double Role for Ascorbic Acid in the Anisotropic Growth of Silver on Gold
}

Kinanti Aliyah ${ }^{1}$, Jieli Lyu ${ }^{1}$, Claire Goldmann ${ }^{1}$, Thomas Bizien ${ }^{2}$, Cyrille Hamon ${ }^{1 *}$, Damien Alloyeau $^{3 *}$, and Doru Constantin ${ }^{1 *}$

${ }^{1}$ Université Paris-Saclay, CNRS, Laboratoire de Physique des Solides, 91405 Orsay, France.

${ }^{2}$ SWING beamline, SOLEIL Synchrotron, Gif-sur-Yvette, France.

${ }^{3}$ Laboratoire Matériaux et Phénomènes Quantiques, Université de Paris - CNRS, F-75013, Paris, France.

\begin{abstract}
Rational nanoparticle design is one of the main goals of materials science, but it can only be achieved via a thorough understanding of the growth process and of the respective roles of the molecular species involved. We demonstrate that a combination of complementary techniques can yield novel information with respect to their individual contributions. We monitored the growth of long aspect ratio silver rods from gold pentatwinned seeds by three in situ techniques (small-angle x-ray scattering, optical extinction spectroscopy and liquid-cell transmission electron microscopy). Exploiting the difference in reaction speed between the bulk synthesis and the nanoparticle formation in the TEM cell, we show that the anisotropic growth is thermodynamically controlled (rather than kinetically) and that ascorbic acid, widely used for its mild reductive properties plays a shape-directing role, by stabilizing the $\{100\}$ facets of the silver cubic lattice, in synergy with the halide ions. This approach can easily be applied to a wide variety of synthesis strategies.
\end{abstract}


Keywords: nanoparticle growth, noble metals, anisotropy, SAXS, in situ liquid transmission electron microscopy

\section{Figure for the table of contents}

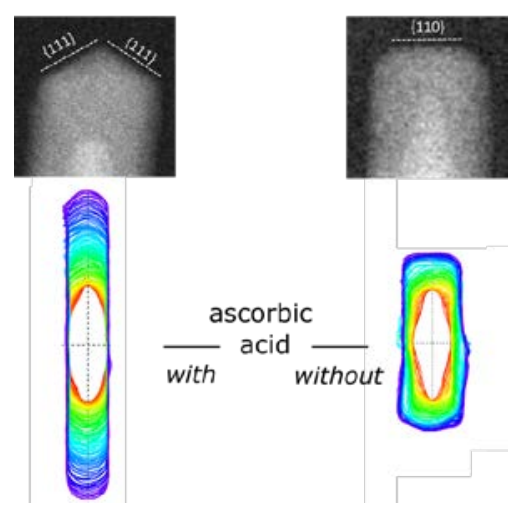

Silver and gold nanostructures are technologically relevant because of their exceptional lightmatter interactions that originates from localized surface plasmon resonances (LSPR). Core@shell Au@Ag nanoparticles (NPs) have recently emerged as a convenient synthetic pathway to obtain anisotropic NPs with well-defined shape and optical properties. ${ }^{1-3}$ Depending on the gold seed crystallinity, the silver shell will grow at different locations, changing the overall morphology of the particle ${ }^{4}$ which results from a competition between the rate of atom deposition and NP faceting driven by possible preferential adsorption sites and surface diffusion, in other words kinetics versus thermodynamics. ${ }^{5-6}$

Experimentally, different NP morphologies can be obtained by varying the deposition rate onto the seeds via reaction parameters such as the $\mathrm{pH}$, the temperature as well as the nature and concentration of reductant and surfactant species. ${ }^{7-10}$ The description of the synthetic pathways of anisotropic Au@Ag NPs has mostly relied on spectroscopy measurements and ex situ TEM. ${ }^{1,}$

${ }^{11}$ Although this approach has greatly expanded the available library of particle shapes and compositions, time-resolved information on the growth mechanisms and reaction intermediates remains limited. 
Diffraction and spectroscopy techniques can be used to follow NP formation in the growth medium, with high temporal resolution. ${ }^{12-16}$ However, given their limited spatial resolution and the necessity to develop models to retrieve the NP morphology, these in situ approaches provide an indirect and incomplete overview of the nucleation and growth processes implied in wetchemical synthesis. Over the last few years, Liquid-Cell Transmission Electron Microscopy (LCTEM) has become a leading method for directly visualizing the dynamics of single nanostructures in their formation medium. ${ }^{17-18}$ An important feature of this technique is that the electron dose rate can be used to control reaction kinetics because NP formation is generally driven by radiolysis processes. ${ }^{19-20}$ This opportunity to control the growth rate and chemical environment of nanostructures has been exploited to reveal the growth mechanisms of various metallic and bimetallic NPs, ${ }^{21-24}$ including core-shell nanostructures. ${ }^{25-30}$ However, exploiting this in situ information in high-yield synthesis remains difficult because radiochemical effects are not involved in conventional protocols. Therefore, an important challenge is to reduce the gap between nanoscale in situ observations and bench-scale synthesis, by correlating LCTEM results with in situ techniques based on light-matter interactions, which probe large NP ensembles without radiolysis effects.

Here, we study the anisotropic growth of silver shells on preformed gold bipyramidal seeds (Figure S1). The seeds are injected in an aqueous growth solution containing silver salt $\left(\mathrm{AgNO}_{3}\right)$, a mild reducing agent (ascorbic acid, AA) and the ionic surfactant cetyltrimethylammonium chloride (CTAC). ${ }^{1,} 31$ The formation of these bimetallic nanostructures was monitored in real time by using multimodal and multiscale in situ measurements (Figure 1). 


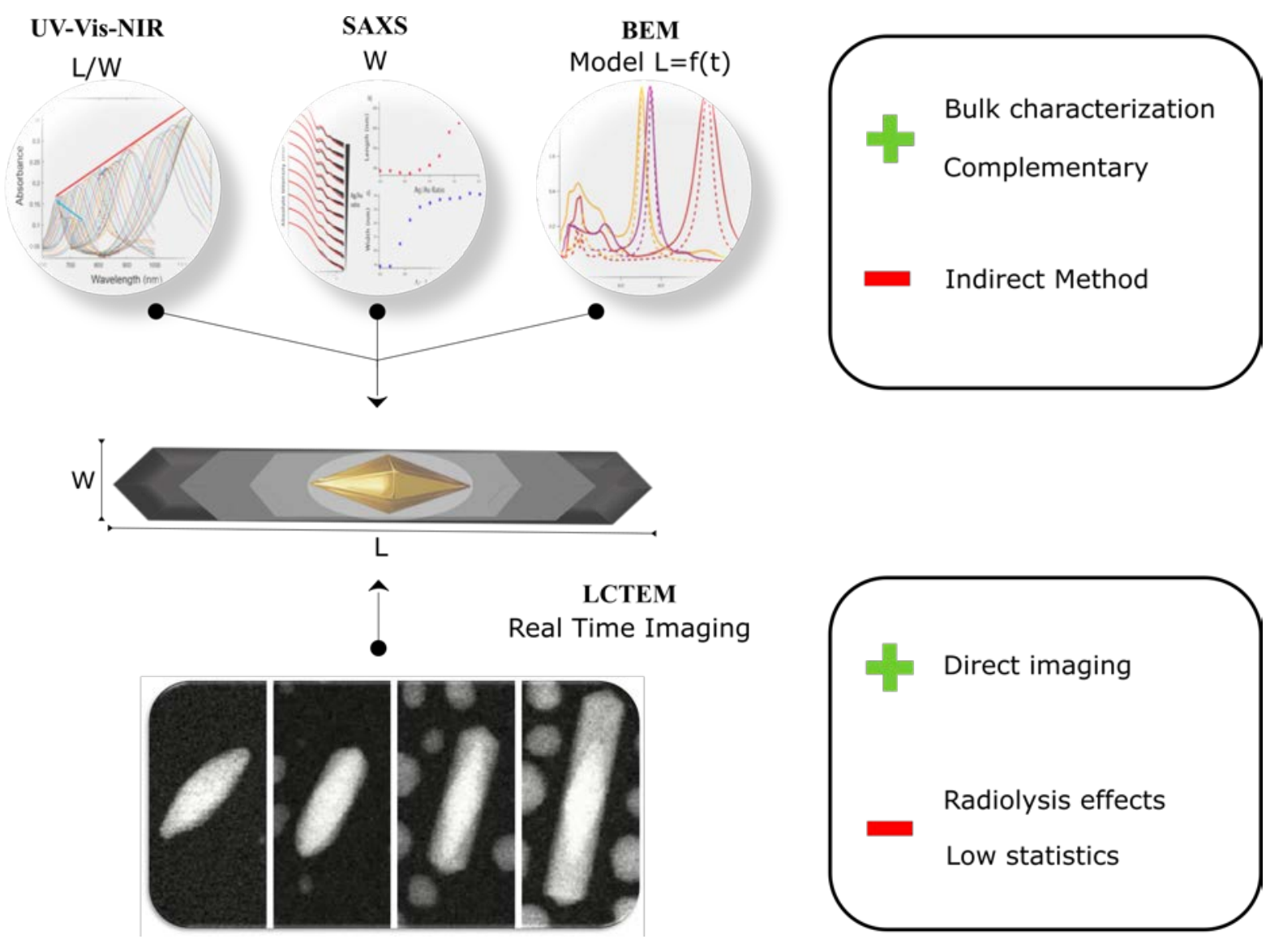

Figure 1: Schematic representation of the methodology for the real time investigation of Au@Ag rod growth. A combination of bulk experiment techniques and numerical modelling in conjunction with direct LCTEM imaging is used to follow the reaction.

LCTEM was exploited to follow the formation of single NPs and confirm the growth pathway inferred from using a combination of in situ Small Angle X-Ray Scattering (SAXS) and UVVis spectroscopy techniques on bulk solutions. In the different experiments, the growth rate differs by more than one order of magnitude, yet yields similar anisotropic nanoproducts, suggesting that the reaction is under thermodynamic control. Furthermore, we show that ascorbic acid is essential to obtain anisotropic bimetallic NPs not only as a source of electron but also as a shape-directing agent. 
Au@Ag rods were synthesized with a high shape yield by controlling silver overgrowth along the fivefold axis of bipyramidal seeds (Figure S2, Figure 2). The final length can be adjusted by varying the silver nitrate precursor $\left(\mathrm{AgNO}_{3}\right)$ amount in the synthesis (see the Supplementary information). In the following, we fixed the chemical composition (i.e. Au seeds, CTAC, AA and $\mathrm{AgNO}_{3}$ ) and the reaction temperature to $60^{\circ} \mathrm{C}$. Ex situ HRTEM analysis of these NPs revealed that the twin planes of the seeds are conserved all along the rods. The silver shells are formed by epitaxial growth on the bipyramidal seeds leading to multi-twinned nanorods with a 5-fold symmetry and a <110> anisotropy axis (Figure S2). The nanorod tips are pentagonal pyramids with a small $\{110\}$ facet at the top and five $\{111\}$ facets (Figure S3). Using the same experimental protocol, we followed the growth of bimetallic NPs in real time by UV-Vis spectroscopy and SAXS. Gold bipyramids seeds exhibit a longitudinal LSPR centered at 752 nm. Upon silver overgrowth, the UV-Vis extinction spectra show a blue shift of this peak by $102 \mathrm{~nm}$ after 9 minutes and a red shift by $512 \mathrm{~nm}$ after 48 minutes, time at which the system stops evolving (Figure 2a and Figure S4). 

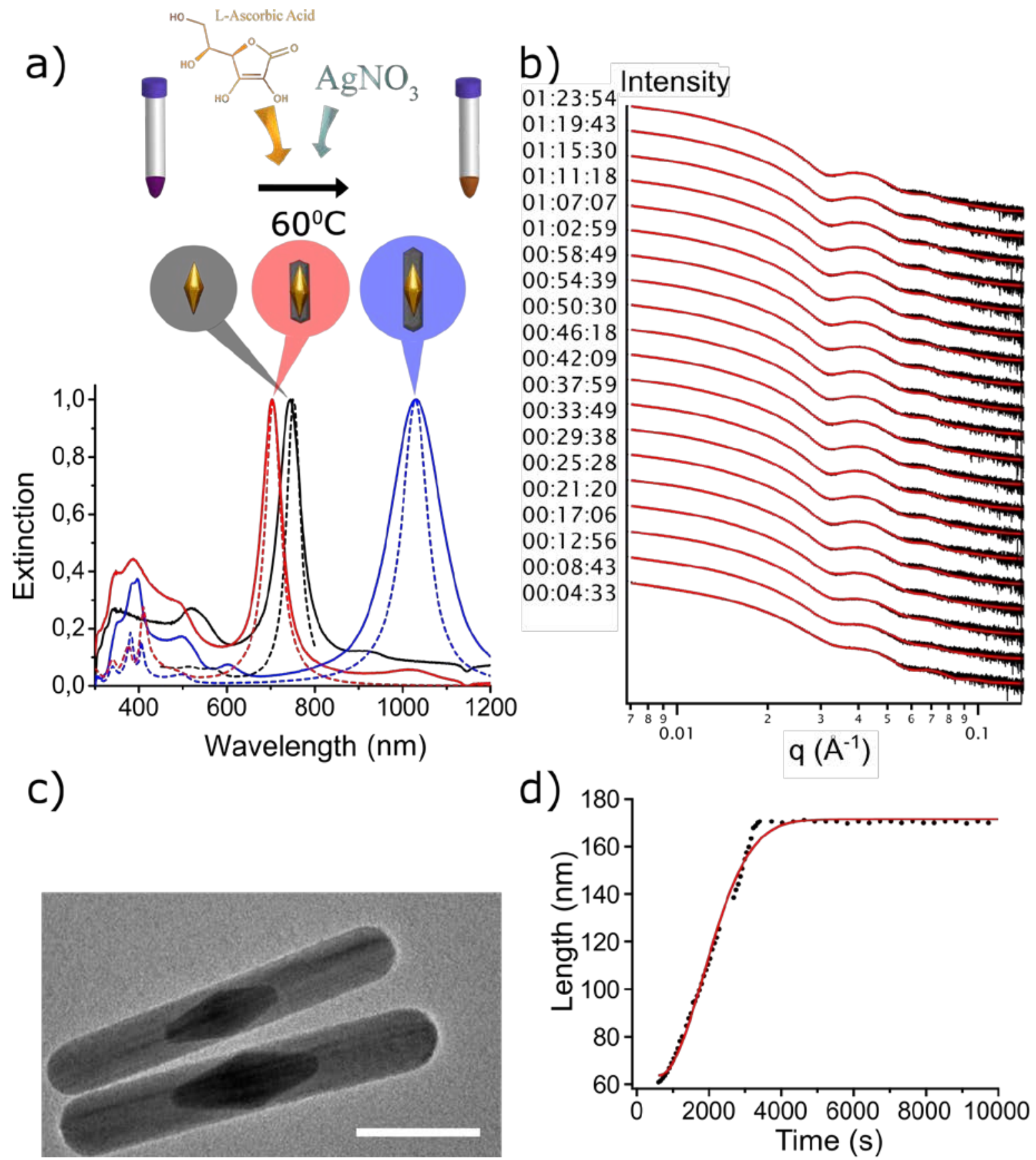

Figure 2: Presentation of the system and ensemble time resolved measurement of Au@Ag rods. a, (top) Scheme depicting the synthesis of Au@Ag rods and the shape transition from a bipyramid to a rod. (bottom) Time-resolved UV-Vis measurement of Au@Ag rods synthesis; for clarity, we show only three spectra, representing three important stages of the reaction: gold bipyramid seeds (black), seeds completely covered in Ag, yielding a short rod (red) and long rod obtained as final result (blue). The dashed lines correspond to BEM models. b, Timeresolved SAXS curves (black) with fits (red) of diluted suspension of Au@Ag rods during their synthesis. Curves are shifted vertically for clarity. c, TEM image of Au@Ag rods obtained in ex situ synthesis. Scale bar is 100 nm. d, Determination of the length of Au@Ag rods as a function of time (black dots) by the combined UV-Vis, SAXS and BEM modelling approach and corresponding fit with the Avrami equation (1) (red line). 
The blue and red shifts correspond to a decrease and increase in aspect ratio, respectively, consistent with the growth of the initial bipyramid into a short rod which then increases in length (see the sketch in Figure 2a). Corresponding SAXS measurements (Figure 2b) describe the evolution of the form factor with oscillations of increasing definition, indicating the formation of particles with a more homogeneous thickness, consistent with a shape transformation from a bipyramid to a rod, with an effective width $W=24.0 \mathrm{~nm}$ (Figure S5). After about 38 minutes, the features no longer changed in the large $q$ range $\left(q>0.025 \AA^{-1}\right)$ while still evolving in the lower $q$ range $\left(q<0.025 \AA^{-1}\right)$, indicating that the objects have a fixed width but an increasing length. The rod length can also be extracted directly from the SAXS curves, ${ }^{32}$ but this method is unreliable for particles longer than the reciprocal of the minimum scattering vector. We use a more precise strategy, obtaining the aspect ratio $L / W$ of the particles from boundary element method (BEM) simulations of the experimental UV-Vis spectrum (Figure 2a and Figure S6). With $W$ obtained from SAXS, $L$ can be better estimated. A rigorous determination of $L(t)$ is essential for understanding the growth kinetics, especially in the case of very anisotropic particles. We model this parameter using a version of the Avrami equation, ${ }^{33}$ with the distinction that, in our case, saturation occurs due to precursor exhaustion, not to the lack of free space:

$$
L(t)=L_{0}+\left(L_{\text {final }}-L_{0}\right)\left\{1-\exp \left(-\left[\beta\left(t-t_{0}\right)\right]^{k}\right)\right\}
$$

Fitting the experimental data with this model (Figure 2d) yields a time constant $\beta=5.710^{-4} \mathrm{~s}^{-1}$ and an exponent $k \cong 2$, consistent with linear growth onto seeds that gradually become active during the reaction (see the Supplementary information for details). 

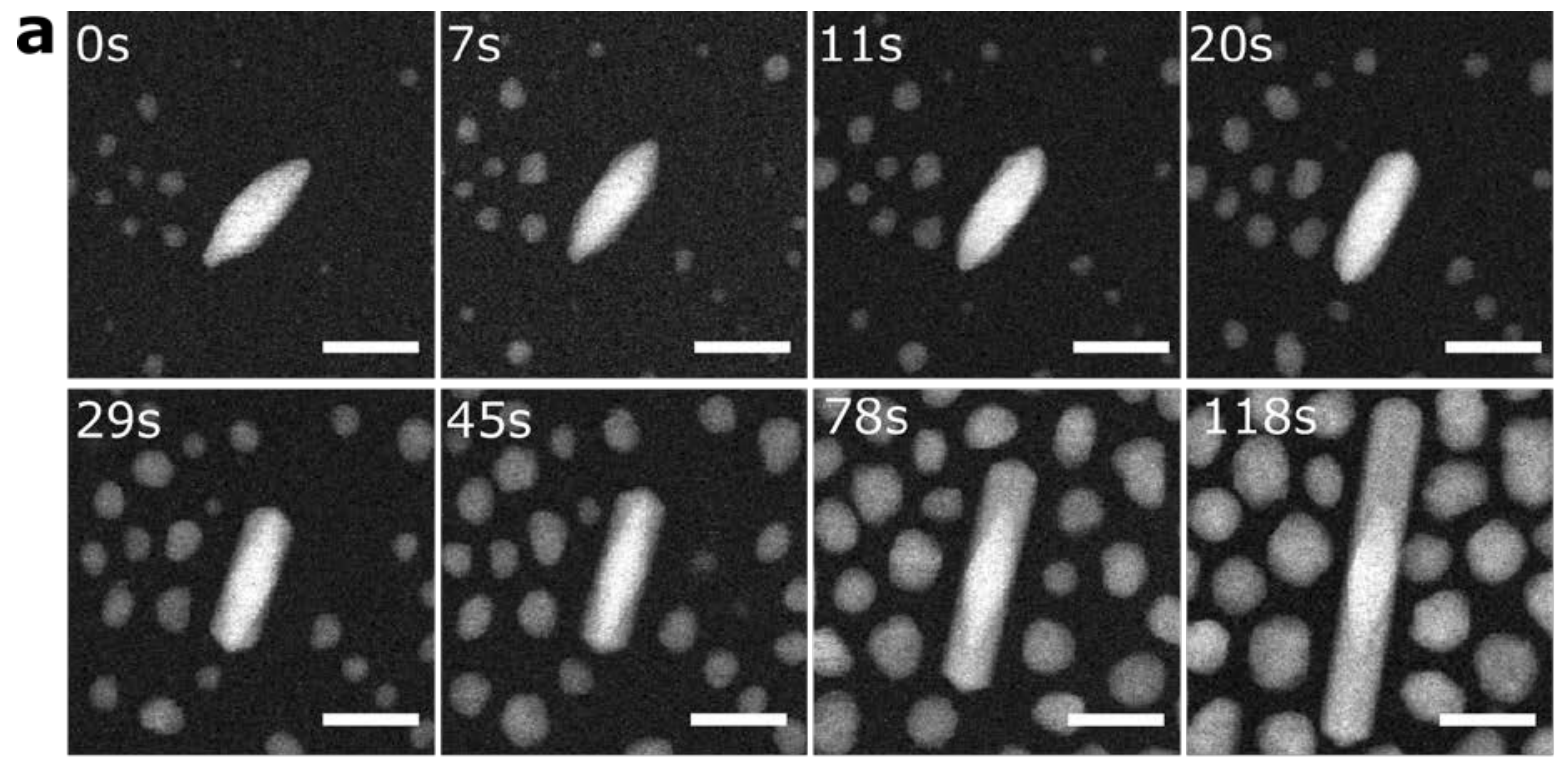

b
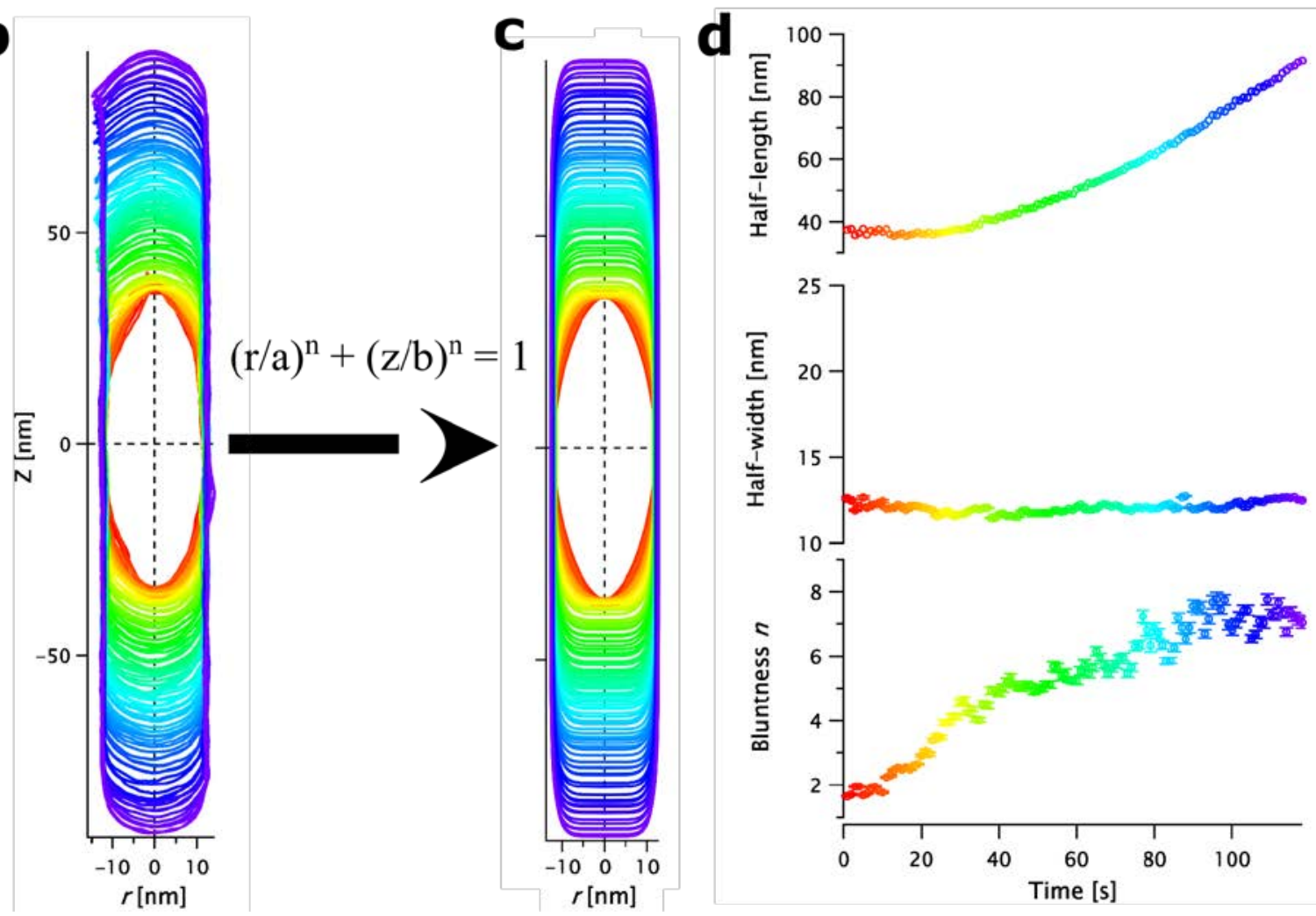

Figure 3: Time-resolved growth of Au@Ag rods by LCTEM. a, Time-lapse STEM images showing the anisotropic deposition of silver on a gold bipyramid as well as homonucleation. Scale bar is $50 \mathrm{~nm}$ on all images. The associated movie (accelerated 16 times) is available as Video_1. b, Contour plots of the Au@Ag rods extracted every second from image analysis. c, Corresponding fit with a superellipsoid model. d, Time dependence of length, width and bluntness of the same Au@Ag rod as determined from the superellipsoid model. The color code of these parameters corresponds to that of the outlines in $\mathbf{b}$ and the fits in $\mathbf{c}$. 
We conducted LCTEM experiments in STEM-HAADF mode to distinguish gold and silver nanocrystals by their difference in Z-contrast (see Supplementary information). An identical reaction mixture to that studied above was confined in the liquid cell and investigated in ambient conditions. Although the reaction rate in bulk synthesis is rather slow at room temperature, bimetallic NPs were obtained within minutes in the irradiated area, confirming that silver reduction is speeded up by radiolysis. The image sequence in Figure 3 shows clearly the anisotropic deposition of silver on the bipyramidal template alongside with the formation of faceted silver NPs that nucleate on the SiN membrane. As many silver-based crystal phases could form under the electron beam, ${ }^{26}$ we exploited the STEM nanodiffraction technique ${ }^{34}$ to confirm that the shells around the gold seeds and the facetted NPs are all made of metallic silver, with a face-centered cubic structure and a lattice parameter of $0.41 \mathrm{~nm}$ (Figure S7). Extracting contour plots of the Au@Ag nanostructure from the entire image series revealed the spatiotemporal evolution of the overgrowth reaction (Figure 3b). These contour plot were fitted with a superellipsoid model: $(r / a)^{n}+(z / b)^{n}=1$, where $r^{2}=x^{2}+y^{2}$ is the radial distance, $z$ is parallel to the long axis of the NP, and parameters $a=W / 2$ and $b=L / 2$ are the half-width and half-length, respectively (Figure 3c). It is well established in the literature that the rods have a pentagonal cross-section in the $(x, y)$ plane, perpendicular to their long axis $z$. For simplicity, we assume this cross-section to be circular; growth in the direction normal to the image is taken as identical to that along the small axis of the object. The parameter $n$ describes the "bluntness" of the particles: $n=1$ describes a diamond, $n=2$ an ellipse and then, as $n$ increases, a less and less rounded rectangle. During the first forty seconds, the sizes $a$ and $b$ remain constant and only the bluntness varies, as the initial bipyramid “fills up” to yield a short rod. As illustrated by Figure 3a (and by the associated video, see the Supplementary materials), this shape transformation goes through the deposition of Ag from the common base of the gold bipyramid toward its two tips. This growth process explains the conservation of the five-fold symmetry of 
the nanostructure, as the $<001>$ edges forming the pentagonal base of the bipyramid generate the five $\{100\}$ side facets of the nanorods. Once the nanostructure reaches a rodlike shape it starts elongating, whereas the increase in width does not exceed $1 \mathrm{~nm}$. It should be noted that systematic discrepancies persist between the experimental particle shapes and the superellipsoid model, which fails to capture the high curvature at the tip of the particles (this morphological detail is quite important, as discussed below). Long-term observation leads to the formation of NRs up to $300 \mathrm{~nm}$ long (Figure S8). Unlike other studies, ${ }^{25,}{ }^{29}$ in our experiments, the growth is mainly governed by monomer attachment. Coalescence events were very seldom observed, generally at the end of the growth process, when the nanorods and the surrounding Ag NPs finally get in contact (Figure S9).

To provide a deeper understanding of this anisotropic growth mode, it is crucial to evaluate kinetic and thermodynamic effects on the shape of NPs. Interestingly, the growth rate of the Au@Ag NPs was very different in our nanoscale and bulk-scale in situ investigations. Indeed, the longitudinal growth rate deduced from UV-Vis spectroscopy and BEM simulations was $0.046 \mathrm{~nm} / \mathrm{s}$ (Figure 2d). We further show that the growth rate of the Au@Ag rods can be varied between 0.0045 and $0.13 \mathrm{~nm} / \mathrm{s}$ by performing the overgrowth reaction at other reaction temperatures and precursor concentrations (Figure S10). In the LCTEM experiments the longitudinal growth rate of the NPs varies from 0.54 to $1.25 \mathrm{~nm} / \mathrm{s}$ (Figure S11a). The radiolysis of water generates several radicular and molecular species including aqueous electrons and hydrogen radicals that are two strong reducing agents. As demonstrated by many groups both experimentally and theoretically, ${ }^{19-20,23-24}$ these radiolytic products can reduce metal precursors and activate the nucleation and growth of metallic nanoparticles within the liquid cell. Therefore, the much higher longitudinal growth rate observed in the liquid cell is very likely due to radiolysis that boosts the silver atom supply. Surprisingly, the growth rate did not scale with the electron dose rate but seems rather dependent on the local availability of silver 
precursor feedstock. The latter is therefore diffusion-limited and most likely affected by screening due to the neighboring silver particles that can occasionally lead to discontinuity of the growth rate over time (Figure S11b).

We emphasize that the identity in mechanism between the bulk synthesis and the LCTEM situation is crucial for our argument. To support this argument we rely on the remarkable similarity in shape evolution between the two environments, cf. Figure 2a and Figure 3 (or the associated Supplementary Movie, Video_1.) In the beginning, the bipyramid "fills up" to yield an ellipse. Remarkably, the lateral expansion of the NPs (along their short axis), both in the bulk synthesis and in LCTEM, is limited to a few atomic layers. In a second step, the particles elongate at constant cross-section. We illustrate the correspondence between the environments in Figure S17, which displays a comparison between the TEM images of particles synthesized at varying $\mathrm{Ag} / \mathrm{Au}$ ratio and images extracted from an LCTEM synthesis.

This absence of kinetic effects on the shape of the NPs indicates that the anisotropic growth along the $<110>$ direction is governed by thermodynamic effects, most likely due to the passivation of the lateral $\{100\}$ facets by capping ligands.

In LCTEM, metallic precursor reduction can be driven by radiolysis alone. We took advantage of this feature to conduct an overgrowth reaction without AA in the reaction medium and reveal the role of this chemical in the anisotropic growth process. 

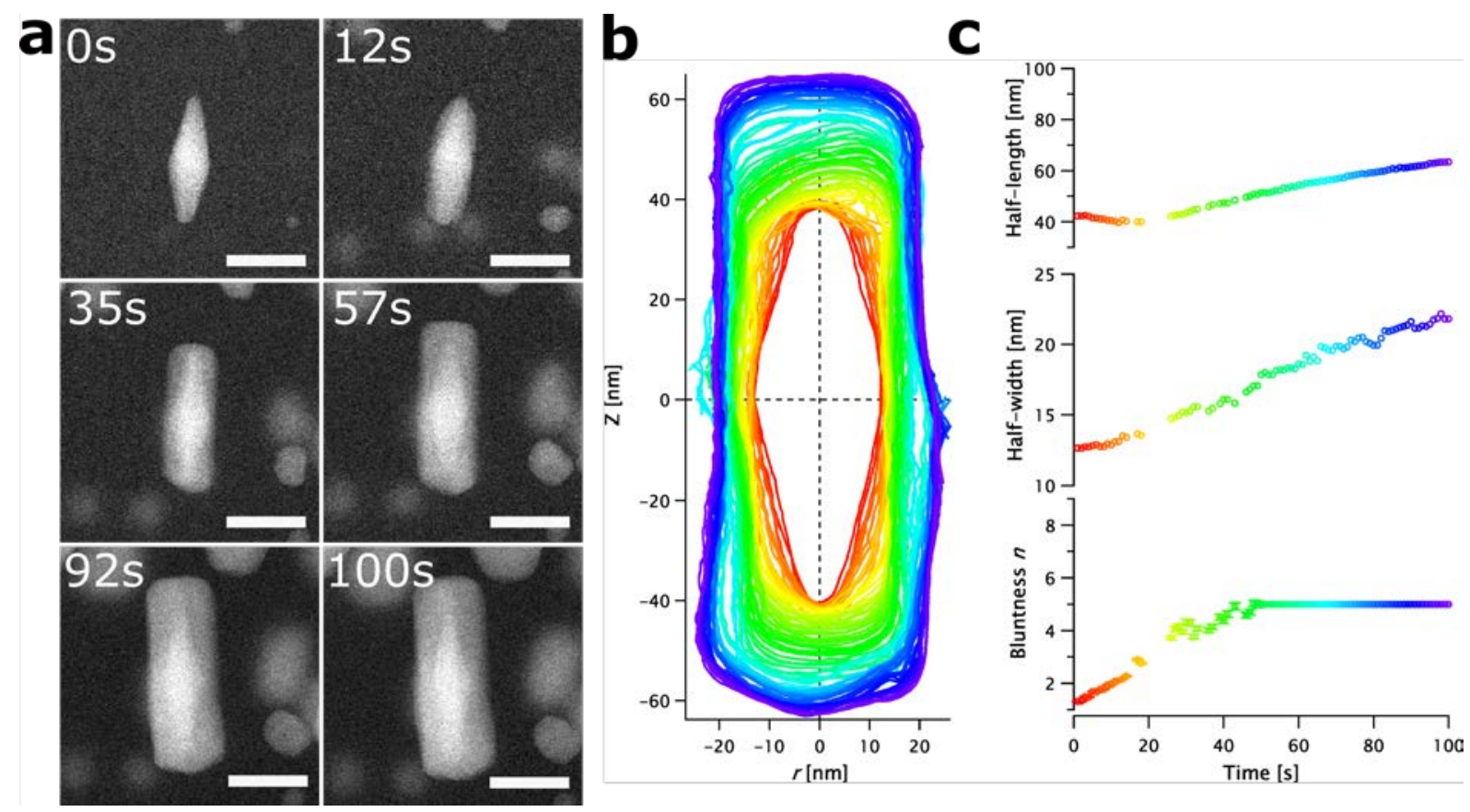

Figure 4: Time-resolved growth of Au@Ag rods by LCTEM, without ascorbic acid. a, Time-lapse STEM images showing the isotropic deposition of silver on a gold bipyramid as well as homonucleation. Scale bar is $50 \mathrm{~nm}$ on all images. The associated movie (accelerated 16 times) is available as Video_2. b, Contour plots of the Au@Ag rods extracted every second from image analysis. The contour plots were first extracted by image analysis and then fitted by a superellipsoid model. c, Graphs show the time dependence of length, width and bluntness of the sameAu@Ag rod as a function of time as determined from the superellipsoid model. The color code of these parameters corresponds to that of the outlines in $\mathbf{b}$.

Figure 4 shows that isotropic silver shells were obtained without AA, suggesting a dramatic modification of the growth pathway. The transformation of bipyramids into nanorods in the first stage of shell formation is similar with and without AA. However, the subsequent growth proceeds at similar speed in the longitudinal and lateral directions $(0.66$ and $0.23 \mathrm{~nm} / \mathrm{s}$, respectively), suggesting an isotropic radial growth in three dimensions (Figure 3c). Other nanorods with almost perfect isotropic growth can be seen in Figure S12. The dose rate range used in the experiments performed with and without ascorbic acid are the same (between 340 and 880 electrons. $\mathrm{s}^{-1} \cdot \mathrm{nm}^{-2}$, depending on magnification). These in situ observations indicates that AA drives the anisotropic growth of silver on gold, but a direct correlation with batch synthesis was needed to support LCTEM results, in particular to ensure that radiolysis effects, such as the possible transformation of AA, do not impact these directional growth processes. In 
order to confirm the role of AA in bulk experiments, halides had to be removed from the reaction medium due to their known role in stabilizing the $\{100\}$ facets of silver. ${ }^{31,35}$ This was challenging because the positively charged headgroups of the surfactant (i.e. $\mathrm{CTA}^{+}$) are balanced with halides counterions (i.e. $\mathrm{Cl}^{-}$). Fortunately, we successfully stabilized the gold bipyramids with sodium polystyrene sulfonate (NaPSS) ${ }^{36}$ and conducted the silver overgrowth reaction in aqueous solution (Figure 5).
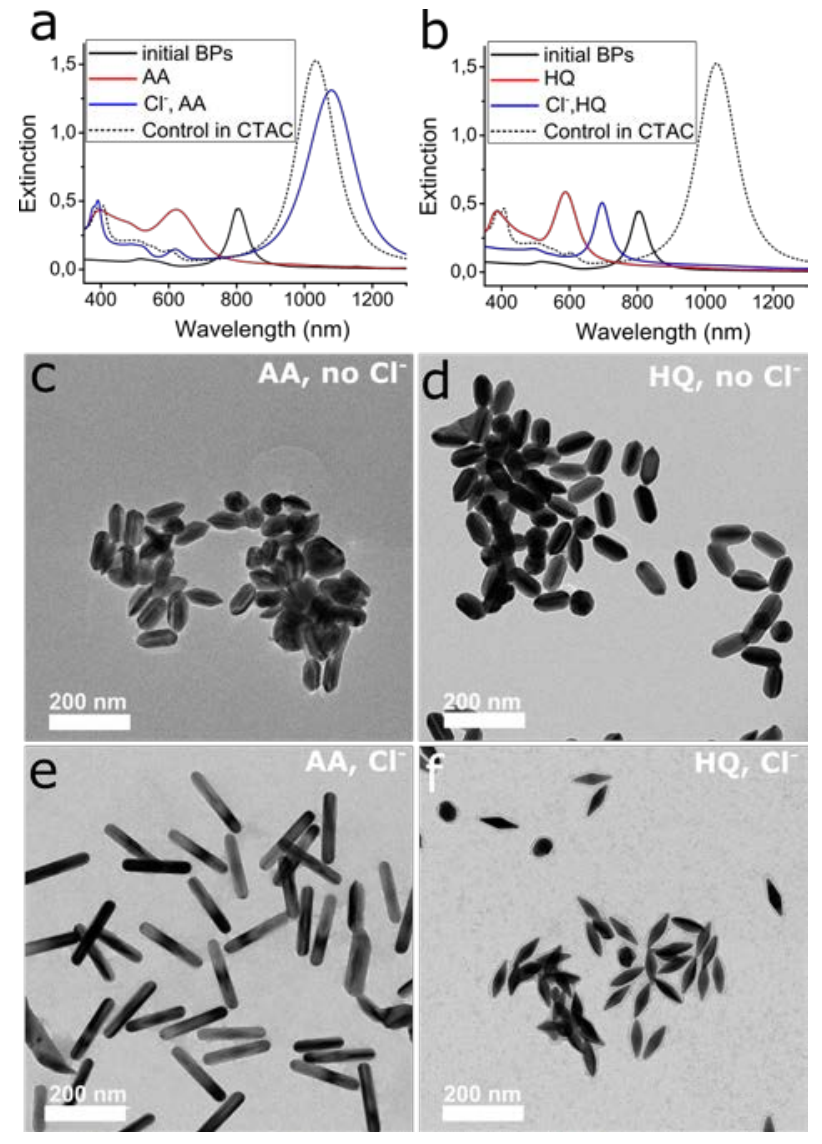

Figure 5: Bulk synthesis of Au@Ag Rods using NaPSS as stabilizer and various reaction conditions. a-b) UV-Vis measurements of the Au@Ag Rods obtained by using AA or hydroquinone (HQ) as reducing agent respectively. Experiments are systematically conducted with (blue solid lines) and without (red solid lines) chlorine ions. References spectra of the gold bipyramids (black solid line) and Au@Rods obtained in CTAC and AA (black dashed line) are displayed for comparison. c-f) Corresponding TEM images.

AA was substituted by hydroquinone (HQ), which was used previously as weak reducing agent in the synthesis of gold nanorods ${ }^{37}$ and we studied the influence of halides by mean of supplementing the reaction medium with $\mathrm{KCl}$ aliquots. First, the reaction was conducted with 
$0.003 \mathrm{M}$ of $\mathrm{AA}$ and varying the concentration of chlorine ions (i.e. trace amounts or $0.01 \mathrm{M}$ ). Remarkably, Au@Ag rods were obtained in the presence of chlorine ions and ascorbic acid, using NaPSS as stabilizer (Figure 5). Although silver deposition was observed without chlorine ions, the growth was not anisotropic in this case. Second, the synthesis was performed in the same way by replacing AA with HQ but we did not observe the formation of Au@Ag rods, even by adding $0.4 \mathrm{M}$ of $\mathrm{HQ}$ in the reaction medium (Figure 5). Halide ions are known to play multiple roles in the synthesis of Au@Ag rods, such as: modifying the Ag redox potential, forming complexes with silver, capping the $\{100\}$ facets and influencing the shape of the surfactant micelles. ${ }^{35}$ Although our results tend to demonstrate that the nature of the surfactant is not relevant in directing the anisotropic growth of silver onto gold bipyramids, we cannot neglect the other possible functions of chlorine ions in the synthesis. Overall, this set of experiments confirms that LCTEM results did not originate in radiolysis effects and confirm the key role of AA, in synergy with chlorine ions, as shape-directing agent in the synthesis of Au@Ag rods.

In this respect, we noticed that AA also has an important impact on the shape of the nanorods' ends during the growth. In the absence of AA the ends are very flat, suggesting the presence of large $\{110\}$ facets that do not favor longitudinal growth. Ultimately, this faceting favors the lateral expansion of the apexes giving a dogbone aspect to the final nanostructures (Figure S13). On the contrary, in the presence of AA, the tips are sharp, forming an angle ranging from $108^{\circ}$ to $125^{\circ}$ (Figure S14) which is consistent with the 2D projections of penta-twinned nanorods with tips terminated by five $\{111\}$ facets, if we consider that their anisotropic axis can slightly deviate from the plane orthogonal to the electron beam. Interestingly, this peculiar tip shape is also observed on NRs obtained in batch synthesis (Figure S3). One can then emphasize that AA also allows maintaining the five-fold symmetry of the NR's tips during the growth. As in decahedral nanoparticles, the farther from the central axis of the multi-twinned nanostructures, 
the greater the strain in the lattice. As suggested by Lofton et al., ${ }^{38}$ this strain distribution could stop the growth along the highly strained (100) facets and favor the elongation of the rods in a direction that does not increase the strain. Such defect-driven growth mechanisms, that result from the symmetry of the bipyramidal seeds, likely contribute to the formation nanorods, but the stabilization of the large (100) facets by chemisorption is also essential to maintain the shape of the nanostructure over time. ${ }^{39}$

In conclusion, our LCTEM investigations confirm the key role of AA in guiding silver-on-gold growth processes. Tan et al. ${ }^{29}$ have recently shown that AA ensures the formation of homogeneous and conformal silver shells on gold nanocubes. By working with anisotropic multi-twinned gold NPs, we confirm that the role of AA in seed mediated synthesis should be reconsidered, as it is clearly an essential parameter to conserve and exploit the symmetry of nanoseeds with complex structure. Commonly described as a mild-reducing agent, we demonstrate here that AA, combined with halides ions, is also a shape-directing agent that favors the formation of nanorods by stopping crystal growth along the $<001>$ directions. This is an important result for future optimization and all applications already using those particles such as sensing ${ }^{40}$ and biomedical technologies. ${ }^{41}$ More generally, the present work shows that such multimodal in situ studies provide a clearer image of the growth mechanisms of nanomaterials. First of all, corroborating large-scale and nanoscale investigations allows us to sidestep the inherent limitations and artifacts associated with each technique. Second, this approach allows determining whether crystal growth is under kinetic or thermodynamic control and finally it provides a way of identifying the individual role of chemicals in the growth mechanisms, helping our understanding of nanomaterial synthesis.

We should emphasize that our result differs from the work of Visaveliya and Köhler ${ }^{42}$, which describes the synthesis of Ag nanowire by a seedless approach, through the polyol process at $160^{\circ} \mathrm{C}$, and it uses PVP as a stabilizer and shape-directing agent. 
These authors also conclude that AA is essential for the anisotropic growth of silver rods, but only consider the reducing role of AA in their study and ascribe to it a kinetic effect (due to an increase in reaction rate) on the formation of multi-twinned particles, which then act as seeds. In our work, we use preformed seeds and demonstrate that AA has a thermodynamic effect (by stabilizing the $\{100\}$ facets).

Our method can readily be extended to other chemicals that are believed to have a double role (e.g. both reducing and shape directing agent). For instance, citric acid is widely used as a reducing agent, but it also appears to stabilize the $\{111\}$ facets of $\mathrm{Pd}^{43}$ and $\mathrm{Au}^{44}$ Experiments ${ }^{45}$ and density functional simulations ${ }^{46}$ indicate that polyvinylpyrrolidone binds more strongly to the $\{100\}$ than to the $\{111\}$ facets of Ag crystals. Our strategy would allow decoupling the two roles of these compounds and hence a thorough analysis of the growth process, as shown above for AA. 


\section{Acknowledgements}

J.L. acknowledges financial supports by the China Scholarship Council (CSC) for her Ph.D. K.A.'s internship was supported by the European Commission under programme Erasmus Mundus Serp-Plus and by the Labex PALM. The SOLEIL synchrotron is acknowledged for the provision of beam time (experiment 20181790, SWING beamline). The present work has benefited from the electronic microscopy facility of Imagerie-Gif, (http://www.i2bc.parissaclay.fr), member of IBiSA (http://www.ibisa.net), supported by “France-BioImaging” (ANR10-INBS-04-01), and the Labex “Saclay Plant Science” (ANR-11-IDEX-0003-02). The CNRS is acknowledged for funding and support. We gratefully acknowledge the financial support of the Region Ile-de-France (convention SESAME E1845) and the Labex SEAM for the JEOL ARM 200F electron microscope installed at the University of Paris exploited for HRTEM and LCTEM experiments.

\section{Author contributions}

KA, JL and CG prepared samples. All authors performed experiments. KA, CH, DA and DC analyzed data. $\mathrm{CH}$, DA and DC wrote the paper with input from all authors, supervised and coordinated the project.

\section{References}

1. Gilroy, K. D.; Ruditskiy, A.; Peng, H. C.; Qin, D.; Xia, Y. Bimetallic Nanocrystals: Syntheses, Properties, and Applications. Chem. Rev. 2016, 116 (18), 10414-72.

2. Gu, J.; Zhang, Y.-W.; Tao, F. Shape control of bimetallic nanocatalysts through welldesigned colloidal chemistry approaches. Chem. Soc. Rev. 2012, 41 (24), 8050-8065.

3. Li, X.; Lyu, J.; Goldmann, C.; Kociak, M.; Constantin, D.; Hamon, C. Plasmonic Oligomers with Tunable Conductive Nanojunctions. J. Phys. Chem. Lett. 2019, 10 (22), 70937099. 
4. Xia, Y.; Gilroy, K. D.; Peng, H. C.; Xia, X. Seed-Mediated Growth of Colloidal Metal Nanocrystals. Angew. Chem. Int. Ed. 2017, 56 (1), 60-95.

5. Xia, Y.; Xia, X.; Peng, H. C. Shape-Controlled Synthesis of Colloidal Metal Nanocrystals: Thermodynamic versus Kinetic Products. J. Am. Chem. Soc. 2015, 137 (25), 7947-66.

6. Polte, J. Fundamental growth principles of colloidal metal nanoparticles - a new perspective. CrystEngComm 2015, 17 (36), 6809-6830.

7. Mayer, M.; Scarabelli, L.; March, K.; Altantzis, T.; Tebbe, M.; Kociak, M.; Bals, S.; García de Abajo, F. J.; Fery, A.; Liz-Marzán, L. M. Controlled Living Nanowire Growth: Precise Control over the Morphology and Optical Properties of AgAuAg Bimetallic Nanowires. Nano Lett. 2015, 15 (8), 5427-5437.

8. $\quad$ Park, K.; Drummy, L. F.; Vaia, R. A. Ag shell morphology on Au nanorod core: role of Ag precursor complex. J. Mater. Chem. 2011, 21 (39), 15608-15618.

9. Okuno, Y.; Nishioka, K.; Kiya, A.; Nakashima, N.; Ishibashi, A.; Niidome, Y. Uniform and controllable preparation of Au-Ag core-shell nanorods using anisotropic silver shell formation on gold nanorods. Nanoscale 2010, 2 (8), 1489-1493.

10. Zhuo, X.; Zhu, X.; Li, Q.; Yang, Z.; Wang, J. Gold Nanobipyramid-Directed Growth of Length-Variable Silver Nanorods with Multipolar Plasmon Resonances. ACS Nano 2015, 9 (7), 7523-35.

11. Huo, D.; Kim, M. J.; Lyu, Z.; Shi, Y.; Wiley, B. J.; Xia, Y. One-Dimensional Metal Nanostructures: From Colloidal Syntheses to Applications. Chem. Rev. 2019, 119(15), 89729073.

12. Abécassis, B.; Testard, F.; Spalla, O.; Barboux, P. Probing in situ the Nucleation and Growth of Gold Nanoparticles by Small-Angle X-ray Scattering. Nano Lett. 2007, 7 (6), 17231727. 
13. Li, T.; Senesi, A. J.; Lee, B. Small Angle X-ray Scattering for Nanoparticle Research. Chem. Rev. 2016, 116 (18), 11128-11180.

14. Polte, J.; Ahner, T. T.; Delissen, F.; Sokolov, S.; Emmerling, F.; Thünemann, A. F.; Kraehnert, R. Mechanism of Gold Nanoparticle Formation in the Classical Citrate Synthesis Method Derived from Coupled In Situ XANES and SAXS Evaluation. J. Am. Chem. Soc. 2010, 132 (4), 1296-1301.

15. Ranasinghe, J. C.; Dikkumbura, A. S.; Hamal, P.; Chen, M.; Khoury, R. A.; Smith, H. T.; Lopata, K.; Haber, L. H. Monitoring the growth dynamics of colloidal gold-silver core-shell nanoparticles using in situ second harmonic generation and extinction spectroscopy. J. Chem. Phys. 2019, 151 (22), 224701.

16. Zhang, Y.; Liu, J.; Ahn, J.; Xiao, T.-H.; Li, Z.-Y.; Qin, D. Observing the Overgrowth of a Second Metal on Silver Cubic Seeds in Solution by Surface-Enhanced Raman Scattering. ACS Nano 2017, 11 (5), 5080-5086.

17. de Jonge, N.; Ross, F. M. Electron microscopy of specimens in liquid. Nat. Nanotechnol. 2011, 6 (11), 695-704.

18. Ross, F. M. Opportunities and challenges in liquid cell electron microscopy. Science 2015, 350 (6267), 1490.

19. Schneider, N. M.; Norton, M. M.; Mendel, B. J.; Grogan, J. M.; Ross, F. M.; Bau, H. H. Electron - Water Interactions and Implications for Liquid Cell Electron Microscopy. J. Phys. Chem. C 2014, 118 (38), 22373-22382.

20. Woehl, T. J.; Abellan, P. Defining the radiation chemistry during liquid cell electron microscopy to enable visualization of nanomaterial growth and degradation dynamics. $J$. Microsc. 2017, 265 (2), 135-147. 
21. Ahmad, N.; Bouar, Y.; Ricolleau, C.; Alloyeau, D. Growth of dendritic nanostructures by liquid-cell transmission electron microscopy: a reflection of the electron-irradiation history. Adv. Struct. Chem. Im. 2016, 2 (1), 9.

22. Ahmad, N.; Wang, G.; Nelayah, J.; Ricolleau, C.; Alloyeau, D. Exploring the Formation of Symmetric Gold Nanostars by Liquid-Cell Transmission Electron Microscopy. Nano Lett. 2017, 17 (7), 4194-4201.

23. Alloyeau, D.; Dachraoui, W.; Javed, Y.; Belkahla, H.; Wang, G.; Lecoq, H.; Ammar, S.; Ersen, O.; Wisnet, A.; Gazeau, F.; Ricolleau, C. Unravelling Kinetic and Thermodynamic Effects on the Growth of Gold Nanoplates by Liquid Transmission Electron Microscopy. Nano Lett. 2015, 15 (4), 2574-2581.

24. Woehl, T. J.; Evans, J. E.; Arslan, I.; Ristenpart, W. D.; Browning, N. D. Direct in Situ Determination of the Mechanisms Controlling Nanoparticle Nucleation and Growth. ACS Nano 2012, 6 (10), 8599-8610.

25. Ahmad, N.; Wang, G.; Nelayah, J.; Ricolleau, C.; Alloyeau, D. Driving reversible redox reactions at solid-liquid interfaces with the electron beam of a transmission electron microscope. J. Microsc 2018, 269 (2), 117-133.

26. Hutzler, A.; Schmutzler, T.; Jank, M. P. M.; Branscheid, R.; Unruh, T.; Spiecker, E.; Frey, L. Unravelling the Mechanisms of Gold-Silver Core-Shell Nanostructure Formation by in Situ TEM Using an Advanced Liquid Cell Design. Nano Lett. 2018, 18 (11), 7222-7229.

27. Jungjohann, K. L.; Bliznakov, S.; Sutter, P. W.; Stach, E. A.; Sutter, E. A. In Situ Liquid Cell Electron Microscopy of the Solution Growth of Au-Pd Core-Shell Nanostructures. Nano Lett. 2013, 13 (6), 2964-2970.

28. Liao, H. G.; Niu, K. Y.; Zheng, H. M. Observation of growth of metal nanoparticles. Chem. Commun. 2013, 49 (100), 11720-11727. 
29. Tan, S. F.; Chee, S. W.; Lin, G.; Bosman, M.; Lin, M.; Mirsaidov, U.; Nijhuis, C. A. Real-Time Imaging of the Formation of Au-Ag Core-Shell Nanoparticles. J. Amer. Chem. Soc. 2016, 138 (16), 5190-5193.

30. Wu, J.; Gao, W.; Wen, J.; Miller, D. J.; Lu, P.; Zuo, J.-M.; Yang, H. Growth of Au on Pt Icosahedral Nanoparticles Revealed by Low-Dose In Situ TEM. Nano Lett. 2015, 15 (4), 2711-2715.

31. Gómez-Graña, S.; Goris, B.; Altantzis, T.; Fernández-López, C.; Carbó-Argibay, E.; Guerrero-Martínez, A.; Almora-Barrios, N.; López, N.; Pastoriza-Santos, I.; Pérez-Juste, J.; Bals, S.; Van Tendeloo, G.; Liz-Marzán, L. M. Au@Ag Nanoparticles: Halides Stabilize \{100\} Facets. J. Phys. Chem. Lett. 2013, 4 (13), 2209-2216.

32. Henkel, A.; Schubert, O.; Plech, A.; Sönnichsen, C. Growth Kinetic of a Rod-Shaped Metal Nanocrystal. J. Phys. Chem. C 2009, 113 (24), 10390-10394.

33. Avrami, M. Kinetics of Phase Change. I General Theory. J. Chem. Phys. 1939, 7 (12), 1103-1112.

34. Khelfa, A.; Byun, C.; Nelayah, J.; Wang, G.; Ricolleau, C.; Alloyeau, D. Structural analysis of single nanoparticles in liquid by low-dose STEM nanodiffraction. Micron 2019, $116,30-35$.

35. Lohse, S. E.; Burrows, N. D.; Scarabelli; Liz-Marzán, L. M.; Murphy, C. J. Anisotropic Noble Metal Nanocrystal Growth: The Role of Halides. Chem. Mater., 2014, 26 (1), 34-43.

36. Mehtala, J. G.; Zemlyanov, D. Y.; Max, J. P.; Kadasala, N.; Zhao, S.; Wei, A. CitrateStabilized Gold Nanorods. Langmuir 2014, 30 (46), 13727-13730.

37. Vigderman, L.; Zubarev, E. R. High-Yield Synthesis of Gold Nanorods with Longitudinal SPR Peak Greater than 1200 nm Using Hydroquinone as a Reducing Agent. Chem. Mater. 2013, 25 (8), 1450-1457. 
38. Lofton, C.; Sigmund, W. Mechanisms Controlling Crystal Habits of Gold and Silver Colloids. Adv. Funct. Mater. 2005, 15 (7), 1197-1208.

39. Xia, Y.; Xiong, Y.; Lim, B.; Skrabalak, S. E. Shape-Controlled Synthesis of Metal Nanocrystals: Simple Chemistry Meets Complex Physics? Angew. Chem. Inter. Ed. 2009, 48 (1), 60-103.

40. Zhu, X.; Zhuo, X.; Li, Q.; Yang, Z.; Wang, J. Gold Nanobipyramid-Supported Silver Nanostructures with Narrow Plasmon Linewidths and Improved Chemical Stability. Adv. Funct. Mater. 2016, 26 (3), 341-352.

41. Lu, G.; De Keersmaecker, H.; Su, L.; Kenens, B.; Rocha, S.; Fron, E.; Chen, C.; Van Dorpe, P.; Mizuno, H.; Hofkens, J.; Hutchison, J. A.; Uji-i, H. Live-Cell SERS Endoscopy Using Plasmonic Nanowire Waveguides. Adv. Mater. 2014, 26 (30), 5124-5128.

42. Visaveliya, N.; Michael Köhler, J. A self-seeding synthesis of Ag microrods of tuned aspect ratio: ascorbic acid plays a key role. Nanotechnology 2013, 24 (34), 345604.

43. Lim, B.; Jiang, M.; Tao, J.; Camargo, P. H. C.; Zhu, Y.; Xia, Y. Shape-Controlled Synthesis of Pd Nanocrystals in Aqueous Solutions. Adv. Funct. Mater. 2009, 19 (2), 189-200. 44. Lim, B.; Kobayashi, H.; Yu, T.; Wang, J.; Kim, M. J.; Li, Z.-Y.; Rycenga, M.; Xia, Y. Synthesis of $\mathrm{Pd}-\mathrm{Au}$ Bimetallic Nanocrystals via Controlled Overgrowth. J. Am. Chem. Soc. 2010, 132 (8), 2506-2507.

45. Sun, Y.; Mayers, B.; Herricks, T.; Xia, Y. Polyol Synthesis of Uniform Silver Nanowires: A Plausible Growth Mechanism and the Supporting Evidence. Nano Lett. 2003, 3 (7), 955-960.

46. Al-Saidi, W. A.; Feng, H.; Fichthorn, K. A. Adsorption of Polyvinylpyrrolidone on Ag Surfaces: Insight into a Structure-Directing Agent. Nano Lett. 2012, 12 (2), 997-1001. 